\title{
DISCRIMINANT ANALYSIS AS A TOOL FOR DETECTING THE ACOUSTIC SIGNALS OF TERMITES COPTOTERMES CURVIGNATHUS (ISOPTERA: RHINOTERMITIDAE)
}

\author{
Muhammad Achirul Nanda ${ }^{1}$, Kudang Boro Seminar ${ }^{1 *}$, Dodi Nandika ${ }^{2}$, Akhiruddin Maddu ${ }^{3}$ \\ ${ }^{1}$ Department of Agricultural Engineering and Biosystem, Faculty of Agricultural Engineering and \\ Technology, Bogor Agricultural University, Bogor 16680, Indonesia \\ ${ }^{2}$ Department of Forest Products, Faculty of Forestry, Bogor Agricultural University, Bogor 16680, \\ Indonesia \\ ${ }^{3}$ Department of Physics, Faculty of Mathematics and Natural Sciences, Bogor Agricultural University, \\ Bogor 16680, Indonesia
}

(Received: June 2017 / Revised: January 2018 / Accepted: April 2018)

\begin{abstract}
Various methods for termite detection have been developed, one of which is purely based on their acoustic signals. However, this method has a weakness, as it is difficult to separate the signals generated by the termites from noise in the environment. A combination of the feature extraction of the acoustic signals and a classification model is expected to overcome this weakness. In this investigation, we inserted 220 subterranean termites Coptotermes curvignathus into pine wood for feeding activity and observed their acoustic signals. In addition, three acoustic features (shortterm energy, entropy and zero moment power) were proposed to recognize the termites' acoustic signals. Subsequently, these features were analyzed and combined with discriminant analysis to produce a robust classification model. According to the numerical results, the integrated discriminant analysis and the acoustic feature in our termite detection system has an accuracy of $83.75 \%$.
\end{abstract}

Keywords: Acoustic signals; Discriminant analysis; Termite detection system

\section{INTRODUCTION}

Termites are social insects that live in colonies and are an extremely destructive pest in destroying wood. It has been reported that termites also attack buildings, furniture and books; according to Nandika et al. (2015), the spread of termites in Indonesia reached $49.9 \%$ of the total land area, with 93.92 million hectares of forests being the natural habitat for termites. There are approximately 2,200 recognized worldwide species of termite, with 200 species occuring in Indonesia. According to Arinana et al. (2016), the subterranean termite Coptotermes curvignathus is responsible for a high intensity of attacks in Indonesia. Moreover, it is also able to make secondary nests in high buildings. Nandika et al. (2015) report that subterranean termite have attacked apartments and hotels up to the 33rd floor in Jakarta, Indonesia. Furthermore, they also destroy trees, resulting in their eventual death. Nandika et al. (2015) estimate that economic losses due to termite attacks on buildings in Indonesia reached 8.7 trillion IDR in 2015. The threat of such attacks is predicted to continue to increase. The initial step to control termite infestation is by using a detection system. However, due to the cryptic behaviour of termites, manual detection of their infestation in wood or wood products is difficult.

\footnotetext{
${ }^{*}$ Corresponding author's email: seminarkudangboro@gmail.com, Tel. +62-8164834625, Fax.+62-2518623203 Permalink/DOI: https://dx.doi.org/10.14716/ijtech.v9i4.455
} 
A basic termite detection system is based on the phenomena which occur when the wood is being infested by termites, such as temperature, humidity, moisture, acoustics and gas. Hence, the termite detection systems which have been developed to date are based on acoustic emission, microwave radar, temperature sensors, measurement of wood moisture content, geophones, Xrays and borescope cameras. Acoustic emission is one of the nondestructive methods that is the most widely applied to detect the existence of termites in wood. Acoustic signals can be produced by simple termite activities; for instance, when termites are feeding and moving in the wood, they generate acoustic signals. Acoustic signals can also be produced by termites as alarm signals through head banging to the wood (Hager \& Kirchner, 2013).

Farkhanda (2013) proposed that biosensor devices could be a combination of several factors, such as temperature, moisture, movement, feeding and behavior of the termites, in the sensing technology. However, no experimental results, especially on termite detection performance using multiple parameters, are reported by Farkhanda. Most researchers have used piezo probe sensors to detect the existence of termites based on acoustic signals (Lewis et al., 2004; Indrayani et al., 2007). On the other hand, there are various alternatives to acoustic sensors to detect termites; for example, geophones, piezo disks, piezo probes, accelerometers, microphones, microwave radar and piezo film (Mankin et al., 2011). Rach et al. (2013) suggest the use of an electret microphone sensor for insect detection within wood, since this type of sensor is more sensitive to vibration. Therefore, our research on termite detection uses an electret microphone sensor. Lewis et al. (2004) used an acoustic sensor (insect detector®, DowAgrosciences) installed on the subsurface of the wood to detect the drywood termite Incitermes minor (Hagen). This technique proved to be effective in reducing background noise, achieving an accuracy of $89.45 \%$. However, the method is destructive, since the wood must be peeled for acoustic sensor installation. This differs from our method, that has been developed based on a non-destructive method.

The improvement of our method for termite detection is based on a mathematical model using the feature of discriminant analysis of acoustic signals. The advantages of discriminant analysis are that the matrix dimension from the observational data can be represented by several principal components, allowing for consideration of all the characteristics, including the interaction of the variables under consideration (Altman, 1968). This method has recently been applied successfully to agricultural problems (Agusta \& Ahmad 2016). Moreover, discriminant analysis can be utilized to differentiate between the acoustic signals generated by termites and those generated otherwise (background noise).

The remainder of the paper is organized as follows. Section 2 presents the methods for data collection, including the feature extraction of the acoustic signals and the discriminant analysis guidelines. Section 3 then explains the development of the classification model and the performance of the proposed system (i.e., accuracy and apparent error). Section 4 presents a detailed discussion of the results and possible future research directions, while some concluding remarks are made in Section 5.

\section{METHODOLOGY}

The subterranean termite Coptotermes curvignathus was obtained from the termite cultivation room in the Termite Laboratory, Faculty of Forestry, Bogor Agricultural University. The pine wood merkusii Jungh et de Vries was selected as the medium for termite infestation. It had a geometrical parameter of $20(\mathrm{l}) \times 9.5(\mathrm{w}) \times 2.5(\mathrm{~h}) \mathrm{cm}$, with a cavity size of $12(\mathrm{l}) \times 6(\mathrm{w}) \times 0.5$ (h) cm. In the experiment, the wood was divided into two groups: infested and uninfested. The infested group was defined as the wood attacked by termites, and the uninfested group was the wood without termites (normal condition). Each group consisted of four samples of pine wood with an initial moisture content of $8.75 \pm 0.05 \%$. We first inserted 220 termites (200 termite- 
workers and 20 termite-soldiers) into the wood for seven days at a room temperature of $28^{\circ} \mathrm{C}$ with $70 \%$ relative humidity. Furthermore, each piece of wood in each group was monitored for acoustic signals ten times.

Figure 1a shows the acoustic signal monitoring of the termites in the wood. The two electret microphone sensors (Itead studio. China), a frequency range of $0.1-10 \mathrm{kHz}$ and sensitivity of -50 $\mathrm{dB}$, were used to obtain the termites' acoustic signals. The sensors were then connected to the Arduino microcontroller (ATmega328P) to convert the analog signals to digital ones. The microcontroller was also connected to the computer (Lenovo ThinkPad X-240) using Arduino IDE software. Therefore, the data detected by the sensors were automatically displayed on the computer. Figure $1 \mathrm{~b}$ shows the placement of the sensor relative to the center point, which was a diagonal intersection between diagonal lines on the top surface the wood under investigation. The distance of the sensors from the center point was $3 \mathrm{~cm}$ to the right and left. This distance was considered safe because it was still in the area of the cavity where the termites were actively performing the attack process.

(a)

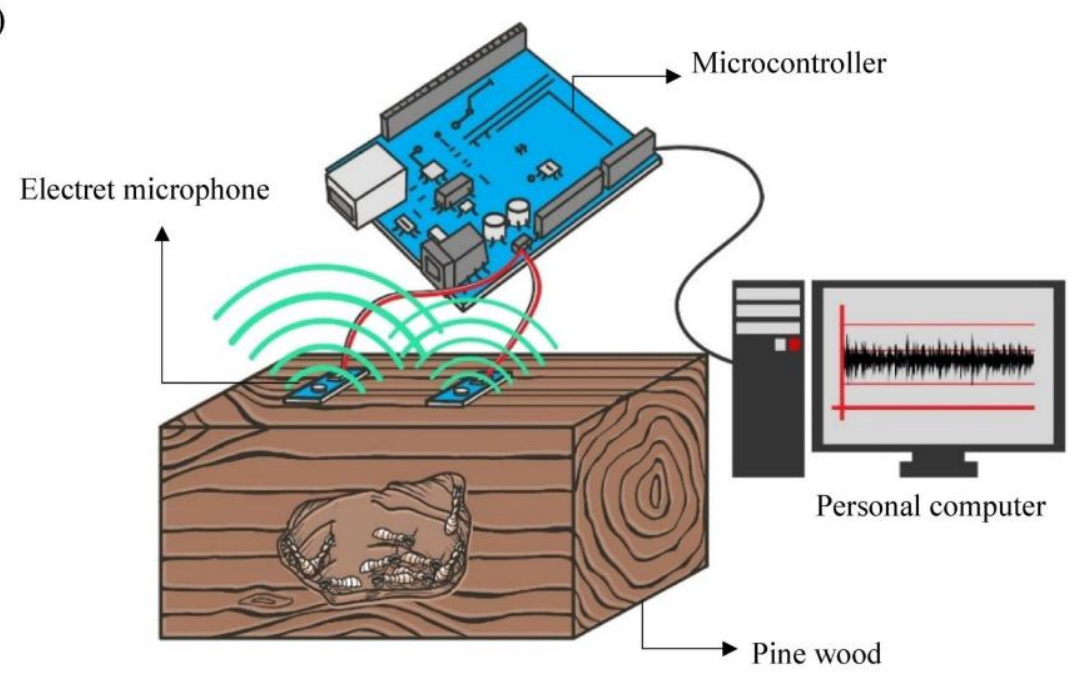

(b)

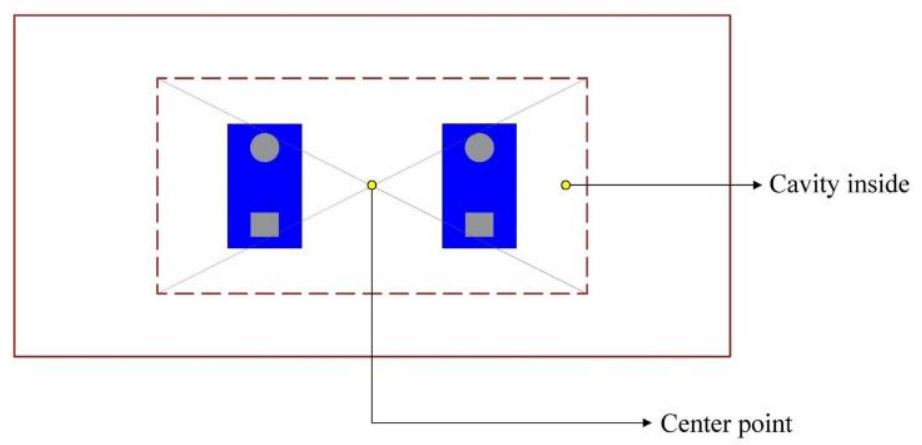

Figure 1 (a) Test apparatus for acoustic signal monitoring of Coptotermes curvignathus; (b) Top view of the sensor placement

\subsection{Acoustic Signal Processing}

In the study, the acoustic signal processing activities are data acquisition, normalization, feature extraction, and classification. First, in the data acquisition process, the acoustic signals produced by the termites were passed through the microcontroller with a sampling rate setting of $100 \mathrm{~Hz}$. To obtain the acoustic signals for one observation, we used a frame size consist of 300 data (within 3 seconds). Second, a normalization process was used to produce clean, ready-to-use data. Third, the feature extraction process was the stage of reducing the data to produce the features that describe the characteristics of the observation object. Figure 2 shows an example of an 
acoustic signal visualization ready to be extracted. Two domain approaches were used to produce features, i.e., time domain (Figure 2a) and frequency domain (Figure 2b). The feature generated in the time domain is a feature obtained without first requiring a signal transformation, meaning it can be directly obtained from the sensors.

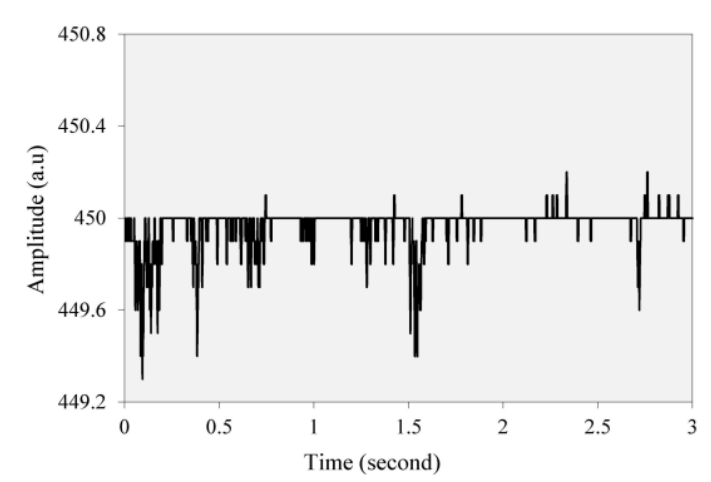

(a)

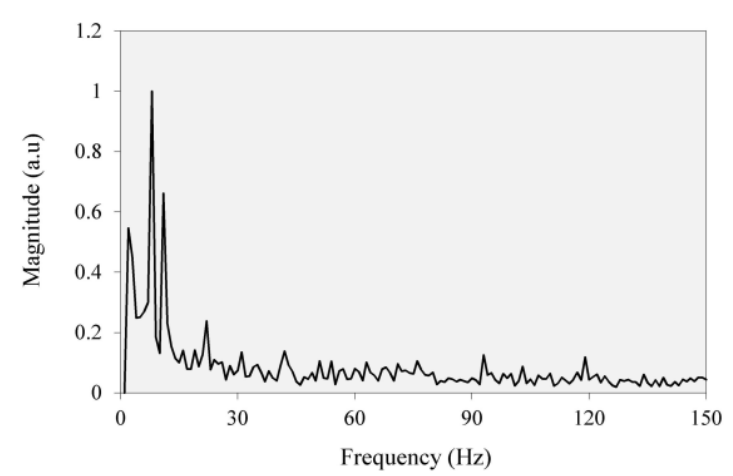

(b)

Figure 2 Acoustic signal visualization ready to be extracted in the: (a) time domain; (b) frequency domain

The features proposed in the time domain as are follows: (a) short-term energy $(E)$, defined as the sum of the squares of the amplitude in the frame. This can be calculated using Equation 1, where $W_{L}=$ frame size, $\left(n=1, \ldots, W_{L}\right)$, and $X=$ amplitude (Nandhini \& Shenbagavalli, 2014; Potamitis et al., 2006); and (b) entropy $(H)$, defined as a measure of abrupt changes in the shortterm energy level from the acoustic signals in the frame. This can be computed using Equation 2 , where $e_{j}$ is the ratio of short-term energy $E(i)$ in the half frame to the frame size (Giannakopoulos \& Pikrakis 2014).

$$
\begin{gathered}
E=\frac{1}{W_{L}} \sum_{n=1}^{W_{L}}|X(n)|^{2} \\
H=-e_{j} \log _{2}\left(e_{j}\right)
\end{gathered}
$$

The feature generated from the frequency domain is a feature that requires signal transformation from the time domain to frequency domain. One of the well-known methods applied to signal transformation is fast fourier transform (FFT). The result of the FFT is given in Figure $2 b$. Visually, it has a significantly different signal shape compared to the signal in the time domain. In this study, the proposed feature of the frequency domain is zero moment power $\left(M_{0}\right)$, which is defined as the area under the peak of magnitude. This can be computed using Equation 3, where $W_{f}=$ frequency length $\left(n=1, \ldots, W_{f}\right), P_{(n)}=$ magnitude and $P_{(n) \max }=$ maximum value of the magnitude.

$$
M_{0}=\sum_{n=1}^{W_{f}} \frac{P_{(n)}}{\left(P_{(n)}\right)_{\max }}
$$

Finally, the classification process recognizes the termite acoustic signals and classifies the infested and uninfested wood. At this stage, we use discriminant analysis to build a classification model.

\subsection{Discriminant Analysis}

Discriminant analysis is a classification model based on multivariate statistical techniques. Classification by discriminant analysis is made because of the interactions of one or more independent variables. In this study, we set the acoustic features (i.e., $E, H, M_{0}$ ) as the independent 
variable. The basic model of discriminant analysis is the linear model, which can be shown in Equation 4 (Uddin et al., 2013), where $d=$ canonical discriminant function, $b_{0}=$ intercept, $b_{n}=$ coefficient, $x_{n}=$ independent variable and $i=1, \ldots, n$.

$$
d=b_{0}+b_{1} x_{1}+b_{2} x_{2}+b_{3} x_{3}+\ldots,+b_{n} x_{n}=b_{0}+\sum_{i=1}^{n} b_{(i)} x_{(i)}
$$

In discriminant analysis, there are three main assumptions which must be fulfilled; if they are not be fulfilled, it will affect the significance and accuracy of the classification result.

\section{Multivariate normal distribution}

In constructing the classification model, the independent variable should follow multivariate normal distribution. This is checked by using the chi-square plot against the mahalanobis distance. This distance can be calculated using Equation 5 (Uddin et al., 2013):

$$
d_{i}^{2}=\left(v_{i}-v\right)^{T} S^{-1}\left(v_{i}-v\right)
$$

where $d_{i}^{2}=$ mahalanobis distance, $T=$ vector to be transposed, $v_{i}=$ the sample vector $(i=1, \ldots, n), v=$ the vector of mean values and $S^{-1}=$ the inverse variance/covariance matrices. Furthermore, if the points follow a straight line pattern of more than $50 \%$ of mahalanobis distance, then it can be ensured that the independent variable follows multivariate normal distribution ( Ramzan et al., 2013; Agusta \& Ahmad, 2016).

\section{No multicollinearity}

Multicollinearity is a statistical phenomenon in which two or more independent variables in multiple regression models are highly correlated to each other. Multicollinearity can be mainly detected with the help of tolerance and its reciprocal, called the variance inflation factor. Tolerance (t) and the variance inflation factor (VIF) are defined by Equations 6 and 7 sequentially (Midi et al., 2010), where $r^{2}$ is the coefficient of determination for the regression of that explanatory variable on all independent variables. Finally, if $t>0.1$ and $V I F<10$, the independent variable is not multicollinear (Akinwande et al., 2015).

$$
\begin{gathered}
t=1-r^{2} \\
V I F=\frac{1}{t}=\frac{1}{1-r^{2}}
\end{gathered}
$$

\section{Homogeneity of variance-covariance matrices}

The assumption in building the classification model using discriminant analysis is that the groups have different variance-covariance matrix homogeneity. This assumption can be tested using Box's M, which transforms the independent variable to an F statistic with df1 and df 2 degrees of freedom. In this test, we set the null hypothesis $\left(\mathrm{H}_{0}\right)$ as

The homogeneity of the variance-covariance matrices of the groups is equal.

If the $p$-value is lower than the significance level $(\alpha)$, then $\mathrm{H}_{0}$ is rejected. As a result, the homogeneity of the variance-covariance matrices of the groups is different.

\subsection{Classification Process}

After all the assumptions are fulfilled, the next step is to build the classification model. First, all the acoustic features of the groups are converted into a principal component using the principal component analysis (PCA) technique. PCA is a statistical method whereby the matrix dimension from the observational data can only be represented by some principal components $\left(\mathrm{PC}_{\mathrm{s}}\right)$, where $s=1,2,3, \ldots, n$ (Ciptohadijoyo et al., 2016). Every PC has cumulative variance; for example, $\mathrm{PC}_{1}$ and $\mathrm{PC}_{2}$ have a cumulative variance of $78 \%$ and $30 \%$ respectively. This means that the $\mathrm{PC}_{1}$ and $\mathrm{PC}_{2}$ can explain the variance in the observational data of $78 \%$ and $30 \%$ sequentially. 
Furthermore, an eigenvalue is used to check the significance of the PC. The minimum acceptable eigenvalue is 1.00; the higher the better (Uddin et al., 2013). Finally, the PC will generate the specific canonical discriminant function.

In this study, the observations of the groups have many scores generated by the canonical discriminant function. To obtain the weight of a particular group, we used the centroid, which can be achieved by calculating the average of the scores. When the centroid is obtained, we also determine the optimal cutting score, which is the weight that shows the separating point between the groups. This can be derived by Equation 8 (Kantardzic, 2011), where, $z_{c s}$ is the optimal cutting score between the groups, $n_{i}$ is the total number observations in the infested group, $n_{u}$ is the total number of observations in the uninfested group, $z_{i}$ is the centroid for the infested group, and $z_{u}$ is the centroid for the uninfested group. After the centroid and the optimal cutting scores are obtained, then the classification model for each group can be produced.

$$
z_{c s}=\frac{n_{i} z_{u}+n_{u} z_{i}}{n_{i}+n_{u}}
$$

\subsection{Performance Assessment}

Before this system can detect automatically whether the wood is infested by termites or not, we need to train the datasets that contain the distinctive acoustic features of a known group. In this study, we used the same data both for training and validating the classification model. To assess the reliability of the model, we used the cross-validation technique, which produces a confusion matrix of $2 \times 2$ size. In Figure 3, the predicted results are the proceeds assessed by the classification model using discriminant analysis, whereas the actual results are the proceeds based on observation (reality). We divided the confusion matrix into four cases: TP, FP, FN, and TN. For example, the TP case is when the actual result and the predicted result reach the same conclusion, i.e., infested. The FP case is when the actual result has an uninfested conclusion, but the predicted result gives an infested conclusion. To calculate the accuracy (AC) and the apparent error (APER) of the predicted results assessed by the classification model, Equations 9 and 10 are used respectively (Rach et al., 2013).

$$
\begin{gathered}
A C=\left(\frac{T P+T N}{T P+F P+F N+T N}\right) 100(\%) \\
A P E R=\left(\frac{F P+F N}{T P+F P+F N+T N}\right) 100(\%)
\end{gathered}
$$

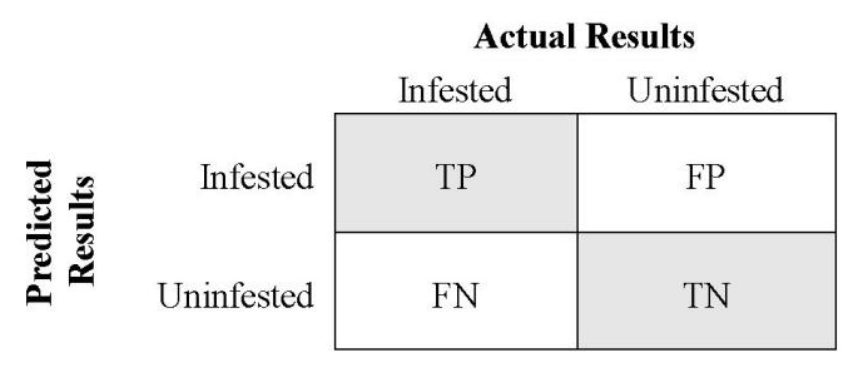

Figure 3 Confusion matrix of discriminant analysis

where:

True positive (TP): correctly classified positive case

False positive (FP): incorrectly classified negative case

False negative (FN): incorrectly classified positive case

True negative (TN): correctly classified negative case 


\section{RESULTS}

\subsection{Acoustic Feature}

We can see in Figure 4 that the infested and uninfested groups can be distinguished according to the performance of $E, H$, and $M_{0}$. Equation 11 is used to find the average value from the distribution of each acoustic feature in a particular group, where $N=$ total number observations of each group, and $\bar{E}, \bar{H}$ and $\bar{M}_{0}$ are the average values of short-term energy, entropy and zero moment power, respectively.

$$
\bar{E}=\frac{1}{N} \sum_{i=1}^{N} E(i), \quad \bar{H}=\frac{1}{N} \sum_{i=1}^{N} H(i), \bar{M}_{0}=\frac{1}{N} \sum_{i=1}^{N} M_{0}(i)
$$

According to the numerical calculation, the infested group has a higher $\bar{E}(0.99926 \pm 0.039)$ than the uninfested group $(\bar{E}=0.99850 \pm 0.042)$. In addition, the value of $\bar{M}_{0}$ from the infested group is 6.65733 \pm 3.11 , which is also greater than that of the uninfested group $\left(\bar{M}_{0}=5.34948 \pm 2.257\right)$. However, the infested group has a lower value of $\bar{H}(-0.30062 \pm 0.034)$ compared to the uninfested group $(-0.30002 \pm 0.024)$. The differences in each characteristic of the acoustic signals feature confirm that there is a significant difference between the normal wood and that infested by the 220 termites, although several observations overlap.

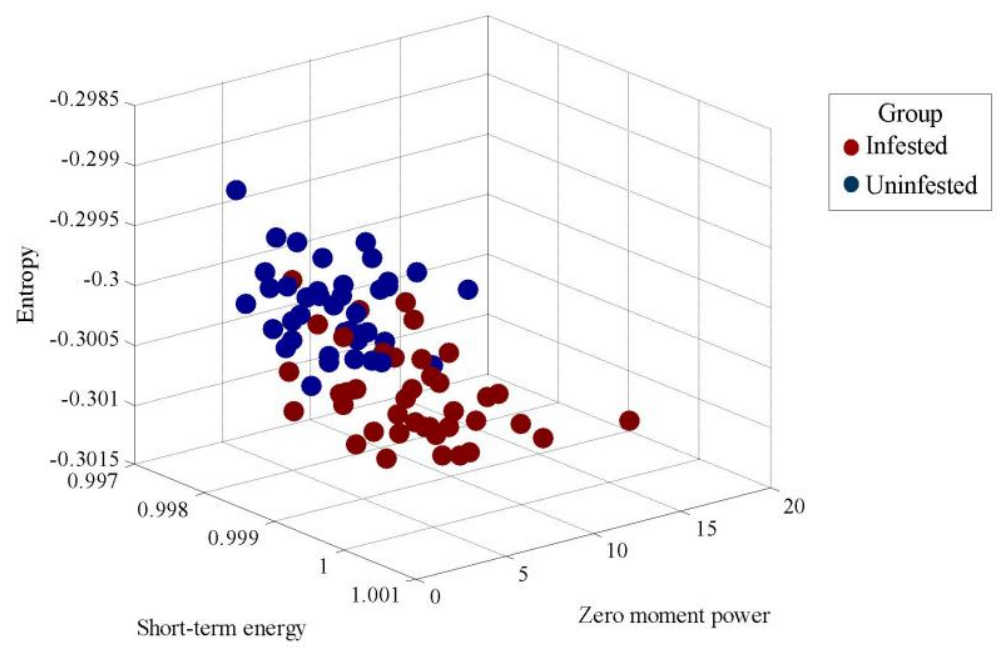

Figure 4 Acoustic feature distribution of the groups

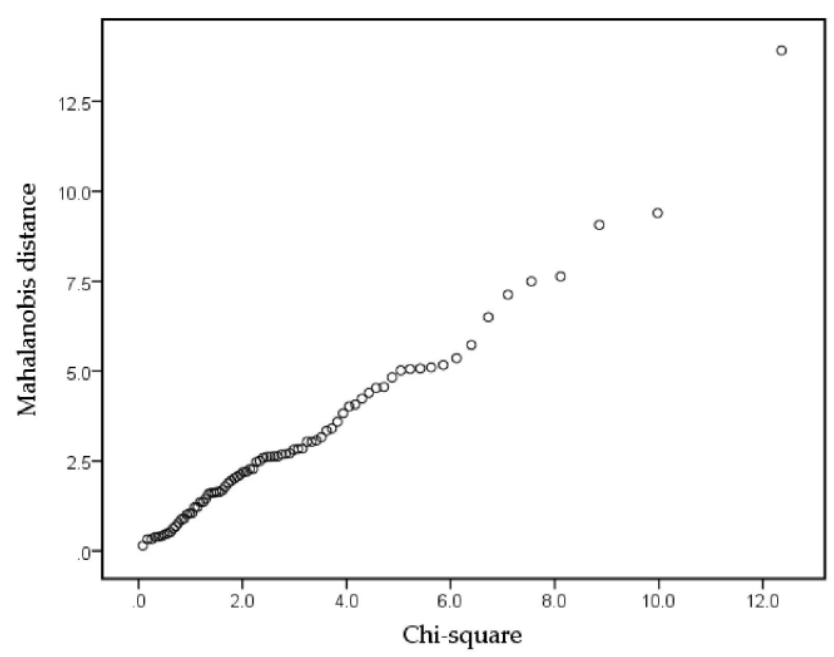

Figure 5 Chi-square plot 


\subsection{Discriminant Analysis Assumptions and Guidelines}

As explained in Section 2.2, before we build the classification model using discriminant analysis, there are three main assumptions which must be achieved. First, to check the multivariate normal distribution, we use the chi-square plot against the mahalanobis distance. As can be seen in Figure 5 , the points follow a straight line pattern of more than $50 \%$ of mahalanobis distance. As a result, the acoustic features (i.e., $E, H$ and $M_{0}$ ) follow multivariate normal distribution.

It is well-known that multicollinearity must be avoided in order to obtain a good statistical model (Midi et al., 2010). According to Table 1, the numerical results of all the acoustic features have $\mathrm{t}>0.1$ and $\mathrm{VIF}<10$, so the acoustic feature is not multicollinear.

Table 1 Multicollinearity testing

\begin{tabular}{cccc}
\hline Statistic & Short-term energy & Entropy & Zero moment power \\
\hline $\mathrm{t}$ & 0.3169 & 0.3077 & 0.8932 \\
$\mathrm{VIF}$ & 3.1553 & 3.2496 & 1.1195 \\
\hline
\end{tabular}

The homogeneity of the variance-covariance matrix test between the infested and uninfested groups can be determined using Box's $M$ test. As can be seen in Table 2, the p-value is less than $\alpha$, i.e., $0.0058(<0.05)$. Therefore, we reject $\mathrm{H}_{0}$. As a result, the homogeneity of the variancecovariance matrices of the groups is different.

Table 2 Results of the homogeneity variance-covariance matrices using Box's M test

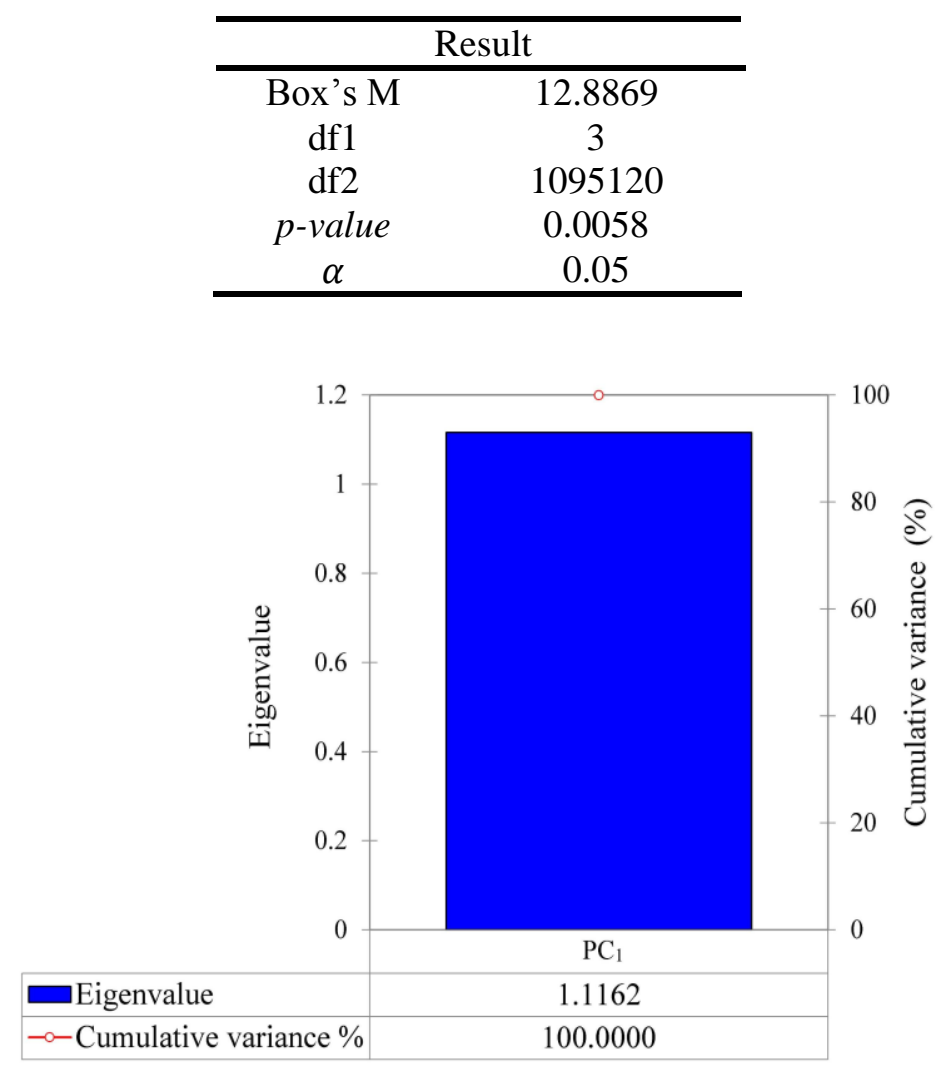

Figure 6 Cumulative variance and eigenvalue of the $\mathrm{PC}_{1}$

\subsection{Classification Process}

\subsubsection{Determination of the canonical discriminant function}

Based on numerical analysis, the PCA technique produces only one PC, namely $\mathrm{PC}_{1}$. As shown in Figure 6, the $\mathrm{PC}_{1}$ has a cumulative variance of $100 \%$, indicating that it can explain $100 \%$ of 
the variance of the observational data. Therefore, the canonical discriminant function will be constructed by the $\mathrm{PC}_{1}$. To check the significance of this, an eigenvalue is used, because the cumulative variance is ordered by its eigenvalue (Rivai \& Tasripan, 2015). According to Figure 6 , the $\mathrm{PC}_{1}$ has a significant eigenvalue, i.e., 1.1162 .

Table 3 shows the parameters of the canonical discriminant function from the $\mathrm{PC}_{1}$. As can be seen, the function has an intercept of -1686.0142 and involves only two acoustic features, $E$ and $H$ (because the coefficient $M_{0}$ is 0 ). These features are 1032.5838 and -2179.5874 respectively. Finally, the canonical discriminant function by $\mathrm{PC}_{1}$ can be written as in Equation 12. In other words, the contributions of this canonical discriminant to the classification model between groups do not overlap.

Table 3 Parameters of the canonical discriminant function generated by $\mathrm{PC}_{1}$

$$
\begin{array}{cc}
\cline { 2 - 2 } \text { Parameter } & \text { Value } \\
\cline { 2 - 2 } \text { Intercept } & -1686.0142 \\
E & 1032.5838 \\
H & -2179.5874 \\
M_{0} & 0 \\
\hline \multicolumn{3}{c}{} \\
d=-1686.0142+1032.5838 E-2179.59874 H
\end{array}
$$

There is an interesting occurrence of the canonical discriminant function, in which the acoustic feature $M_{0}$ is 0 . This means that the feature is not used in the classification model development. Although at the beginning of the process all features meet all the assumptions, we cannot guarantee that all the features will be used. This is a result of the DA itself; optimization is still done through a statistical approach, such as identification of Wilks' lamda value in order to select the relevant features (Uddin et al. 2013). This is an advantage of DA in producing a robust model.

\subsubsection{Classification model}

As shown in Figure 7a, each group has many scores generated by the canonical discriminant function. In this study, two centroids are used to classify the particular group, i.e., that of the infested and uninfested groups. According to the numerical results shown in Figure $7 \mathrm{~b}$, the centroids of the infested and uninfested groups are 1.0432 and -1.0432 , respectively.

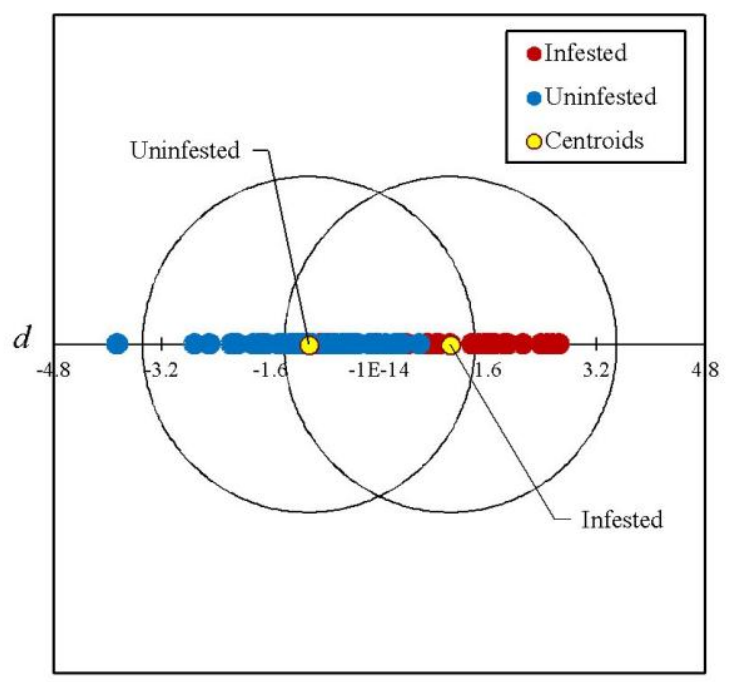

(a)

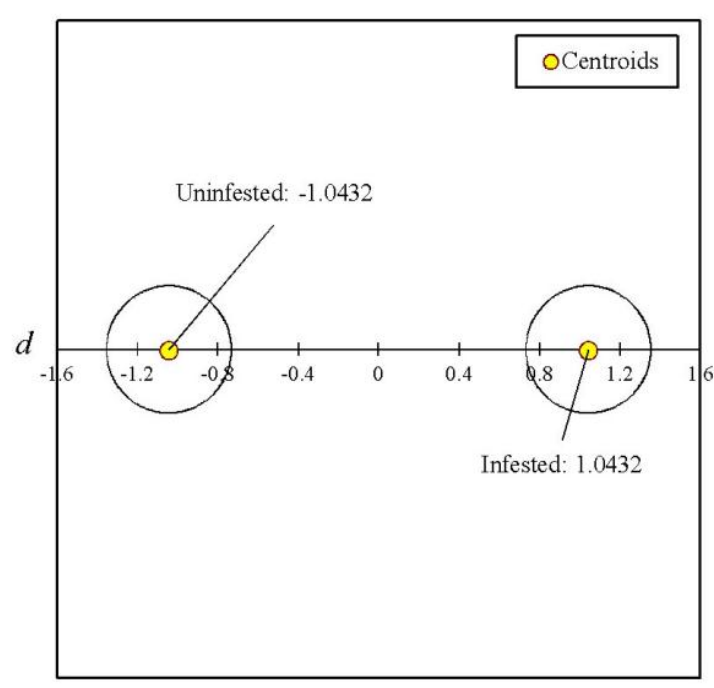

(b)

Figure 7 (a) Plot of scores to classify groups; (b) Centroids of the groups 
Accordingly, the optimal cutting score of both groups is zero. Finally, the classification models of the infested group $(I G)$ and the uninfested group $(U G)$ are derived in Equations 13 and 14, respectively.

$$
\begin{aligned}
& I G=-3213122.7853+7524125.8260 E+3633565.4725 H \\
& U G=-3209605.0053+7521971.3942 E+3638113.0671 H
\end{aligned}
$$

\subsection{Performance of Validation}

Figure 8 shows the confusion matrix results between the actual and predicted results. The numerical results of cases TP, FP, FN, and TN are 33, 7, 6, and 34, respectively. Furthermore, according to the numerical calculation, the classification model embedded in the termite detection system has an accuracy of $83.75 \%$ and an apparent error of $16.25 \%$.

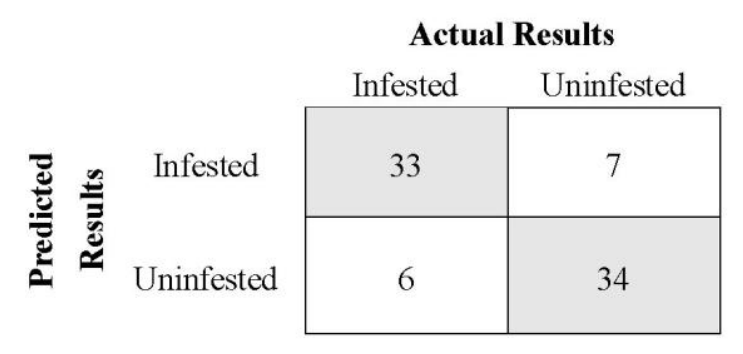

Figure 8 Confusion matrix from the validation

\section{DISCUSSION}

In 2001, termite control in Indonesia developed rapidly, as shown by the establishment of more than 151 companies, especially for controlling termite attacks, and the registering of more than 32 termiticide trademarks (Nandika et al., 2015). One of the ways to control termites is by spraying termiticide on the wood surfaces infested by termites. However, before this control is carried out, the wood should be initially checked to determine whether termites are already inside the wood. To avoid higher damage, detecting termites as early as possible is very important.

Termite acoustic signals are one of the most widely used phenomena in the development of termite detection systems. Our results indicate significant differences in the acoustic features of the infested group and the uninfested group. Termite activity generates acoustic signals, such as from feeding, moving and head banging on the wood. Research explains that termites bang their heads on the wood as a mode of communication, known as vibrational alarm communication (Lehrer, 2013). This habit is performed by all termites, both termite workers and soldiers. This alarm is transmitted inside the nest and the gallery system at a distance of several meters (Lehrer, 2013). In the literature, the vibration alarm signal is generally $<2 \mathrm{kHz}$ and acoustic emission with the ultrasonic signal is $>60 \mathrm{kHz}$ (Evans et al., 2005).

The acoustic features used to build the classification model in our termite detection system were short-term energy and entropy. Both features were obtained in the time domain, which certainly eases computational complexity; when compared with the frequency domain, which first requires signal transformation, short-term energy is one of the feature extractions commonly used in acoustic signals processing. This feature has been successfully applied to solve problems in agriculture, such as detection of the red palm weevil within wood (Hussein et al., 2010); the cricket family (Potamitis et al., 2006); and beetle larvae (Schofield, 2011).

In this investigation, our termite detection system, which has $83.75 \%$ accuracy, was built on two classification models, i.e. Equations 13 and 14. Both models were developed using discriminant analysis. The rule of the models is that we classify the groups (i.e., $I G$ or $U G$ ) corresponding to the classification model that gives the greatest value. For example, if $I G>U G$, then the observation is classified into $I G$. Moreover, these classification models can be used to classify 
new observations into pre-existing groups. These findings indicate that discriminant analysis can be implemented in termite detection systems. However, the type of wood also plays a significant role, affecting the behavior of termites and related production of acoustic signals (Lewis et al., 2004). Therefore, in future studies various types of woods should be investigated to obtain comprehensive information about the performance of our termite detection system.

\section{CONCLUSION}

This study has successfully demonstrated a classification model based on discriminant analysis in a termite detection system. In the selection of the principal component process, short-term energy and entropy were the acoustic features used to build the classification model of the groups. According to the numerical results, the performance of the proposed termite detection system using discriminant analysis has an accuracy and apparent error of $83.75 \%$ and $16.25 \%$ respectively. These findings indicate that the combination of discriminant analysis and feature extraction of the acoustic signals can be integrated into termite detection systems.

\section{ACKNOWLEDGEMENT}

The authors would like to acknowledge the Ministry of Research, Technology and Higher Education of the Public Indonesia through the accelerated master program leading to doctoral research grants (PMDSU) 2016, contract no: 330/SP2H/LT/DRPM/IX/2016.

\section{REFERENCES}

Agusta, W., Ahmad, U., 2016. Study on Golden Apollo Melon Ripeness Level Using Acoustic Impulse Parameters (Mempelajari Tingkat Kematangan Buah Melon Golden Apollo Menggunakan Parameter Sinyal Suara). Jurnal Keteknikan Pertanian, Volume 4(2), pp. 195-202

Akinwande, M.O., Dikko, H.G., Samson, A., 2015. Variance Inflation Factor: as a Condition for the Inclusion of Suppressor Variable (S) in Regression Analysis. Open Journal of Statistics, Volume 5(7), pp. 754-767

Altman, E.I., 1968. Financial Ratios, Discriminant Analysis and the Prediction of Corporate Bankruptcy. The Journal of Finance, Volume 23(4), pp. 589-609

Arinana, A., Aldina, R., Nandika, D., Rauf, A., Harahap, I.S., Sumertajaya, I., Bahtiar, E.T., 2016. Termite Diversity in Urban Landscape, South Jakarta, Indonesia. Insects, Volume 7(20), pp. $1-18$

Ciptohadijoyo, S., Litananda, W., Rivai, M., Purnomo, M., 2016. Electronic Nose based on Partition Column Integrated with Gas Sensor for Fruit Identification and Classification. Computers and Electronics in Agriculture, Volume 121(2016), pp. 429-435

Evans, T.A., Lai, J.C., Toledano, E., McDowall, L., Rakotonarivo, S., Lenz, M., 2005. Termites Assess Wood Size by using Vibration Signals. In: Proceedings of the National Academy of Sciences of the United States of America, Germany. Volume 102(10), pp. 3732-3737

Farkhanda, M., 2013. Biosensors for Termite Control. IOP Conference Series: Materials Science and Engineering, Volume 51, pp. 1-3

Giannakopoulos, T., Pikrakis, A., 2014. Introduction to Audio Analysis: a Matlab® Approach. Academic Press, Oxford, USA

Hager, F.A., Kirchner, W.H., 2013. Vibrational Long-distance Communication in the Termites Macrotermes Natalensis and Odontotermes Sp. Journal of Experimental Biology, Volume 216(17), pp. 3249-3256

Hussein, W.B., Hussein, M.A., Becker, T., 2010. Detection of the Red Palm Weevil Rhynchophorus Ferrugineus using Its Bioacoustics Features. Bioacoustics, Volume 19(3), pp. 177-194 
Indrayani, Y., Yoshimura, T., Yanase, Y., Fujii, Y., Imamura, Y., 2007. Evaluation of the Temperature and Relative Humidity Preferences of the Western Dry-wood Termite Incisitermes Minor (Hagen) using Acoustic Emission (AE) Monitoring. Journal of Wood Science, Volume 53(1), pp. 76-79

Kantardzic, M., 2011. Data mining: Concepts, Models, Methods, and Algorithms. John Wiley \& Sons, Inc, New Jersey, USA

Lehrer, M., 2013. Orientation and Communication in Arthropods. Springer Basel AG, Birkhäuser, Basel, Swiss

Lewis, V.R., Power, A.B., Haverty, M.I., 2004. Surface and Subsurface Sensor Performance in Acoustically Detecting Western Drywood Termites in Naturally Infested Boards. Forest Products Journal, Volume 54(6), pp. 57-62

Mankin, R., Hagstrum, D., Smith, M., Roda, A., Kairo, M., 2011. Perspective and Promise: a Century of Insect Acoustic Detection and Monitoring. American Entomologist, Volume 57(1), pp. 30-44

Midi, H., Sarkar, S.K., Rana, S., 2010. Collinearity Diagnostics of Binary Logistic Regression Model. Journal of Interdisciplinary Mathematics, Volume 13(3), pp. 253-267

Nandhini, S., Shenbagavalli, A., 2014. Voiced/Unvoiced Detection using Short Term Processing. In: Proceedings of the International Conference on Innovations in Information, Embedded and Communication Systems, November, Chennai, India. Volume 4(2), pp. 39-43

Nandika, D., Rismayadi, Y., Diba, F., 2015. Termite: Biology and Its Control, $2^{\text {nd }}$ Edition (Rayap: Biologi dan Pengendaliannya, Edisi Ke-2), Muhammadiyah University Press, Surakarta, Indonesia (in Bahasa)

Potamitis, I., Ganchev, T., Fakotakis, N., 2006. Automatic Acoustic Identification of Insects Inspired by the Speaker Recognition Paradigm. In: Proceedings of the Proc. of the InterSpeech, Pittsburgh, PA, USA. pp. 2126-2129

Rach, M.M., Gomis, H.M., Granado, O.L., Malumbres, M.P., Campoy, A.M., Martín, J.J.S., 2013. On the Design of a Bioacoustic Sensor for the Early Detection of the Red Palm Weevil. Sensors, Volume 13(2), pp. 1706-1729

Ramzan, S., Zahid, F.M., Ramzan, S., 2013. Evaluating Multivariate Normality: A Graphical Approach. Middle East Journal of Scientific Research, Volume 13(2), pp. 254-263

Rivai, M., Tasripan, 2015. Fuel Qualification using Quartz Sensors. ARPN Journal of Engineering and Applied Sciences, Volume 10(16), pp. 6737-6743

Schofield, J., 2011. Real-Time Acoustic Identification of Invasive Wood-Boring Beetles. PhD Thesis, Graduate Program, University of York, York, England

Uddin, N., Meah, M.S., Hossain, R., 2013. Discriminant Analysis as an Aid to Human Resource Selection and Human Resource Turnover Minimization Decisions. International Journal of Business and Management, Volume 8(17), pp. 153-169 